\title{
Estudio Descriptivo Macro y Mesoscópico de la Irrigación de Piezas Dentarias Superiores en Perro (Canis familiaris) Mediante Repleción con Tinta China
}

\author{
Macro and Mesoscopical Study About the Irrigation of the Upper Teeth in Dogs \\ (Canis familiaris) through Repletion With Chinese Ink
}

"Cintya Borroni; ",** Ismael Concha; **Luis Córdova \& ***inaldo Soto

BORRONI, C.; CONCHA, I.; CóRDOVA, L. \& SOTO, R. Estudio descriptivo macro y mesoscópico de la irrigación de piezas dentarias superiores en perro (Canis familiaris) mediante repleción con tinta china. Int. J. Morphol., 24(4):555-559, 2006.

RESUMEN: Se describe macro y mesoscópicamente la irrigación de piezas dentarias superiores en su tercio rostral (incisivos y canino) en perro, mediante repleción con tinta china. Se utilizaron 30 cabezas de cadáveres de perro, divididas en tres grupos de igual número. El grupo I fue inyectado con tinta china azul vía arteria facial, el grupo II con tinta amarilla vía arteria maxilar y el grupo III con tinta azul y amarilla vía arteria facial y arteria maxilar respectivamente y en forma simultánea. En las cabezas, ya fijadas y conservadas, se analizó macroscópicamente la llegada del color de la tinta china inyectada a nivel de la gíngiva asociada a dientes incisivos y canino superior derecho. Posteriormente, las cabezas se congelaron para realizarles cortes transversales, los que fueron analizados mesoscópicamente, donde se observó la llegada de la tinta china, a nivel de los alvéolos y cavidad pulpar de las piezas dentarias en estudio.

La presente investigación demostró que la arteria facial se distribuye hacia tejidos profundos como gíngiva, alvéolos y pulpa de los dientes incisivos y canino superior derecho en perro, por lo tanto, estaría aportando a su irrigación complementaria; se reafirmó la participación de ramas de la arteria maxilar en la irrigación de estos mismos tejidos y finalmente se demostró que ramas de las arterias facial y maxilar se anastomosan para irrigar tejidos en común, como los ya mencionados. Estos resultados permiten conocer el origen de la revascularización de piezas dentarias superiores y periodonto en perro, luego de procedimientos quirúr gicos que pudiesen dañar ramas de la arteria maxilar. Debido a la similitud anatómica de la cavidad oral entre el perro y el humano, los resultados descritos se podrían utilizar como referencia para explicar el origen de la revascularización de piezas dentarias superiores y periodonto, luego de la cirugía ortognática.

PALABRAS CLAVE: Arteria facial; Arteria maxilar; Alvéolo; Cavidad pulpar.

\section{INTRODUCCIÓN}

La corrección de las deformidades en el tercio medio facial, se realiza mediante la cirugía ortognática. Una de estas cirugías consiste en realizar una osteotomía de los huesos maxilares, tipo Le Fort I, para desplazarlos a una nueva posición. El procedimiento implica la sección de los huesos maxilares, dañando las ramas arteriales dentarias provenientes de la arteria maxilar que se encargan de la irrigación de las piezas dentarias superiores. La evidencia clínica indica que no existe necrosis pulpar después de la cirugía ortognática (Cheung, 1998), a pesar que en el post-operatorio inmediato disminuye el flujo sanguíneo hacia la cavidad pulpar; ésta presenta una recuperación creciente a las pocas semanas, siendo completa aproximadamente a los tres meses (Justus et al., 2001). Se desconocen objetivamente los pedículos, a partir de los cuales se produce la revascularización de dichas pulpas dentarias.

En el perro y en humano, el aporte nutricio primario hacia piezas dentarias superiores proviene de ramas alveolares originadas desde la arteria maxilar y la irrigación de la mucosa de los labios nace a partir de la arteria facial (Evans, 1993, Figún \& Garino, 2002). Debido a dichas similitudes, en el presente trabajo se utilizó el perro como un posible modelo anatómico para explicar el origen de la revascularización de piezas dentarias superiores después de la cirugía ortognática. Se describió la distribución de la arteria facial y arteria maxilar a nivel de la gíngiva, alvéolos y pulpa de dientes incisivos y canino superior derecho, en el perro.

\footnotetext{
* Anatomía Veterinaria Universidad Santo Tomás, Sede Santiago, Chile.

** Anatomía Normal Universidad de Los Andes, Chile.

Estudio financiado por Fondo de Tesis de Grado de la Universidad Santo Tomás, Chile.
} 


\section{MATERIAL Y MÉTODO}

Se utilizaron 30 cadáveres de perros mestizos, de tamaños mediano y grande, de ambos sexos, mayores de un año, con todas sus piezas dentarias permanentes y con la cabeza en buenas condiciones. Los perros fueron sometidos a eutanasia debido a enfermedades terminales, y donados por clínicas veterinarias.

Las 30 cabezas obtenidas de los cadáveres, fueron separadas en 3 grupos de 10, con el fin de realizar repleciones con tinta china vía arteria facial, arteria maxilar y por ambas arterias en forma simultánea.

La parte práctica del estudio se realizó en el Laboratorio de la Unidad de Anatomía Veterinaria de la Universidad Santo Tomás, Sede Santiago, y el Laboratorio de Anatomía Normal de la Universidad de los Andes, ambos ubicados en la Comuna de San Bernardo, Chile.

Se registraron 18 cadáveres machos y 12 hembras, con un rango de edad aproximado entre 1 a 10 años determinado por cronología dentaria y un rango de peso aproximado entre 10-40 kilos.

Grupo I: A cada uno de los diez cadáveres de este grupo se les realizó una incisión en la región cervical lateral derecha, fue canulada la vena yugular externa con una bajada de suero, y la arteria carótida común derecha con una sonda de alimentación $\mathrm{N}^{\mathrm{o}} 8$; se procedió al lavado vascular con agua tibia y posteriormente se ligaron estos vasos.

Se realizó una disección de la región hasta ubicar la arterias carótidas común, interna y externa y sus ramas, las que fueron ligadas, a excepción de la arteria facial que fue canulada con una bránula de 18 o 21 G según el diámetro del vaso, para luego inyectar por medio de una jeringa de $10 \mathrm{ml}$, entre $6-10 \mathrm{ml}$ de tinta china color azul, dependiendo del tamaño del perro. Inmediatamente después, se observó el territorio de irrigación de la arteria facial teñido de azul.

Finalizada la repleción, se ligó la arteria facial para evitar el reflujo de la tinta. Se separó la cabeza del resto del cuerpo mediante la desarticulación del atlas y axis. La cabeza fue fijada y conservada mediante inmersión en solución fijadora-conservadora, durante una semana.

Luego de la fijación, las cabezas fueron extraídas de la solución y lavadas con agua. La mucosa oral fue impregnada con agua oxigenada para dar mayor contraste a la repleción y estudiar macroscópicamente el territorio abarcado por la arteria facial a nivel de la gíngiva del arco dentario superior.

Luego, las cabezas fueron congeladas a $-24^{\circ} \mathrm{C}$. Se realizaron siete cortes transversales con una sierra eléctrica vertical, desde los dientes incisivos superiores hasta los dientes premolares superiores número cuatro, aproximadamente, dando énfasis a la cavidad pulpar de dientes incisivos y caninos superiores.

Los cortes transversales fueron sumergidos en agua oxigenada por aproximadamente una hora para aumentar el contraste de la tinta. Para facilitar el acceso a la cavidad pulpar se utilizaron distintos tipos de brocas, fresas y discos para cortar. Cada uno de los cortes fue mantenido en refrigeración entre 0 a $4{ }^{\circ} \mathrm{C}$.

Para el análisis mesoscópico los cortes de cada cabeza se estudiaron bajo una lupa estereoscópica. Se analizó la presencia o ausencia de capilares azules provenientes de la arteria facial a nivel de la cavidad pulpar de dientes incisivos y canino superior derecho, además de la participación de la análisis mesoscópico facial en la irrigación de los alvéolos de estas piezas dentarias.

Grupo II. Para este grupo se utilizó un método similar al anteriormente descrito. En estas cabezas se ligó la arteria facial y se canuló con una sonda de alimentación la arteria maxilar, de mayor diámetro, con una jeringa de $20 \mathrm{ml}$ se inyectó tinta china de color amarillo entre 10 a $15 \mathrm{ml}$ dependiendo del tamaño del perro. Iinmediatamente después de la repleción se observó el territorio de la arteria maxilar teñido de amarillo a nivel del vestíbulo oral.

Las cabezas ya conservadas fueron analizadas macroscópicamente a nivel de la gíngiva asociada al arco dentario superior en su tercio rostral. En el análisis mesoscópico se observó la llegada del color inyectado proveniente de la arteria maxilar a nivel de la pulpa de incisivos y canino superior derecho y sus alvéolos.

Grupo III. Siguiendo con un procedimiento similar a lo descrito para los otros grupos, se ligaron las ramas de la arteria carótida externa a excepción de las arterias facial y maxilar. La arteria facial fue canulada para su posterior inyección de 6-10 ml de tinta china color azul, simultáneamente se inyectó la arteria maxilar, ya canulada, con 10-15 ml de tinta china amarilla. Luego de la repleción, se buscó una mezcla de colores, es decir, que la mucosa del vestíbulo oral presentara color verde, hecho que demostraría que las dos arterias presentan territorios de irrigación en común.

Las cabezas conservadas se impregnaron con agua oxigenada, para su posterior análisis macroscópico de la gíngiva asociada al arco dentario superior en su tercio rostral. En el análisis mesoscópico, se observó la presencia de color verde (mezcla de colores inyectados) a nivel de alvéolos y cavidad pulpar de incisivos y canino superior derecho, esto indicaría que ramas de las arterias facial y maxilar se anastomosan para irrigar tejidos en común. 


\section{RESULTADOS}

Grupo I. Resultados de la evaluación macroscópica de las diez cabezas del grupo I, correspondiente a los territorios irrigados por la arteria facial a nivel de la gíngiva asociada a los incisivos superiores derechos, presentó tinción de color azul en un $10 \%$ de las cabezas muestreadas, a nivel del diente canino derecho se distribuyó en un $80 \%$.

La descripción mesoscópica demostró que la arteria facial se distribuye hacia los alvéolos de los dientes incisivos central, medio, lateral y canino superior derechos en un $90 \%$ de las muestras. A nivel de la cavidad pulpar se observó que un $40 \%$ los dientes incisivo central y medio derecho presentaron tinción azul a nivel de su cavidad, para el incisivo lateral un $50 \%$ y en el diente canino derecho un $90 \%$ de las muestras.

Grupo II. La evaluación macroscópica de las diez cabezas del grupo II, correspondiente a la participación de la arteria maxilar a nivel de la gíngiva asociada a los dientes incisivo central y medio dio como resultado una coloración amarilla en un $60 \%$ de las cabezas, a nivel del incisivo lateral un $70 \%$ y el diente canino, en un $80 \%$.

A nivel mesoscópico, la tinta china amarilla se distribuyó hacia los alvéolos de los dientes incisivo central y medio en un $90 \%$, al incisivo lateral y diente canino superior derecho en un $100 \%$ de las muestras. A nivel de la cavidad pulpar del diente incisivo central, la tinta llegó en un $10 \%$ de las muestras, al incisivo medio, un $30 \%$, al incisivo lateral, un $40 \%$ y al diente canino derecho un $30 \%$. Se esperaba un mayor porcentaje de muestras que presentaran distribución de la tinta china hacia la cavidad pulpar de las piezas dentarias en estudio; esto se puede atribuir a posibles problemas de técnica, basándose en lo descrito por la literatura (Evans) y en que los cadáveres utilizados para este grupo estaban dentro de un rango de edad y peso similares (factores utilizados en todas las muestras de este estudio), entre los cuales, la congelación y posterior descongelación de algunos cadáveres inyectados con tinta china y la presencia de coágulos en la sangre evitarían el flujo normal de la tinta a través de las ramas arteriales, principalmente hacia la cavidad pulpar.

Grupo III. La evaluación macroscópica de la irrigación de la gíngiva de los dientes incisivos central y medio destacó la coloración verde proveniente de la anastomosis entre las arterias facial y maxilar, en un $50 \%$ de las cabezas muestreadas para este grupo, mientras que la gíngiva del incisivo lateral se presentó en el $60 \%$ de las cabezasy para el diente canino en el $70 \%$ de ellas.

A nivel mesoscópico, la coloración verde se presentó
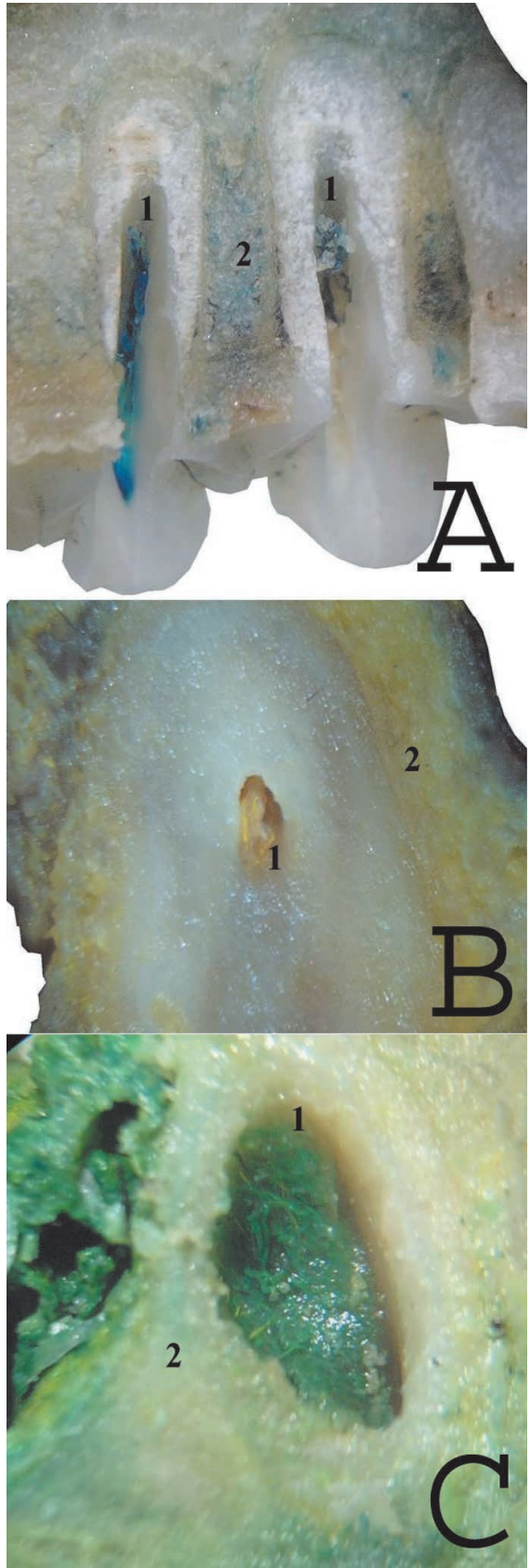

Fig.1A. Corte transversal a nivel de los dientes incisivos central y medio superior derecho. Se observa la presencia de capilares teñidos de color azul provenientes de la arteria facial, a nivel de la cavidad pulpar (1) y alvéolos (2)

Fig. 1B. Corte transversal a nivel de la raíz del diente canino superior derecho. Se observa la presencia de capilares teñidos de color amarillo provenientes de la arteria maxilar, a nivel de la cavidad pulpar (1) y alvéolo (2).

Fig. 1C. Corte transversal a nivel de la raíz del diente canino superior derecho. Se observa la presencia de capilares teñidos de color verde provenientes de la anastomosis entre la arterias facial $y$ maxilar, a nivel de la cavidad pulpar (1) y alvéolo (2).

en el alvéolo del incisivo central derecho en un $90 \%$ de las muestras, mientras que los incisivos medio, lateral y diente canino se observó en el $100 \%$.

A nivel de la cavidad pulpar del diente incisivo central, un $20 \%$ de las muestras presentó coloración verde, el incisivo medio fue de $70 \%$, el incisivo lateral en el $50 \%$ y el diente canino en el $70 \%$. 


\section{DISCUSIÓN}

El estudio macroscópico del grupo I demostró el aporte de la arteria facial en la irrigación de la gíngiva asociada al diente canino superior derecho en mayor porcentaje. El análisis mesoscópico de los alvéolos asociados a los dientes incisivos y canino superior derecho demostró un aporte importante de la arteria facial a su irrigación; esta arteria se distribuye también hacia la cavidad pulpar del diente canino y en menor grado a los incisivos, lo que explicaría por el diámetro mayor del agujero apical del diente canino, lo que permitiría un flujo mayor de tinta china hacia su cavidad pulpar. La literatura menciona que la arteria facial nutre sólo tejidos superficiales, tales como, musculatura y mucosa del labio superior, y son ramas de la arteria maxilar las que aportan la irrigación hacia la gíngiva, alvéolos y pulpa de dientes superiores (Evans), lo que se contrapone a los resultados encontrados en este estudio, que demostró una irrigación complementaria de la arteria facial hacia territorios más profundos, como los anteriormente mencionados.

Los resultados de la evaluación macroscópica de la gíngiva y mesoscópica de los alvéolos asociados a los dientes incisivos y canino superior derecho del grupo II, corroboraron lo descrito en la literatura (Evans), ya que más de la mitad de las muestras presentó color amarillo a nivel de la gíngiva y en mayor proporción la tinta se distribuyó hacia los alvéolos. En el análisis mesoscópico de la cavidad pulpar de las piezas dentarias en estudio, se observó que menos de la mitad de las muestras presentó la pulpa teñida de color amarillo.

La evaluación macroscópica del grupo III demostró que entre las arterias facial y maxilar existe anastomosis, lo que permitiría irrigar la gíngiva asociada a los dientes incisivos y canino superior derecho. Más de la mitad de las muestras presentaron esta tendencia. En el análisis mesoscópico de los alvéolos, se observó color verde (mezcla de colores) a nivel de todas las piezas dentarias estudiadas. En la cavidad pulpar, la coloración se presentó en más de la mitad de las muestras en el incisivo medio, lateral y diente canino, en el incisivo central fue sólo en dos cabezas, debido a que posee un agujero apical más pequeño. Estos resultados demostraron la anastomosis entre ramas de las arteria facial y maxilar, que permitiría la irrigación en común de territorios profundos. Sin embargo, la literatura describe que estas arterias se unen sólo para nutrir tejidos superficiales, tal como la mucosa del labio superior (Evans).

Los hallazgos observados en este trabajo demostraron que la arteria facial aporta irrigación complementaria, por sí sola y mediante anastomosis con la arteria maxilar, hacia tejidos profundos como la gíngiva, alvéolos y pulpa asociada a los dientes incisivos y canino superior. Esto es de vital importancia ya que aseguraría el flujo sanguíneo hacia dientes superiores y periodonto después de procedimientos quirúrgicos realizados en perro, tales como, reparación de fracturas de huesos maxilares o extracción de tumores de la zona, que pudiesen dañar ramas de la arteria maxilar.

Debido a la similitud anatómica de la cavidad oral del perro y del humano, los resultados descritos se podrían utilizar como referencia para explicar el origen de la revascularización de piezas dentarias superiores y periodonto, luego de la sección de ramas alveolares provenientes de la arteria maxilar durante la cirugía ortognática realizada en humanos.

BORRONI, C.; CONCHA, I.; CÓRDOVA, L. \& SOTO, R. Macro and mesoscopical study about the irrigation of the upper teeth in dogs (Canis familiaris) through repletion with chinese ink. Int. J. Morphol., 24(4):555-559, 2006.

SUMMARY: The irrigation of upper teeth is described macro and mesoscopically in their facial third (incisive and canine teeth) through repletion with Chinese tint. Thirty heads of corpses of dogs were used, which were divided in three equal groups. Group I was injected with blue Chinese ink via facial artery, group II with yellow Chinese ink via maxillary artery, and the group III with blue and yellow Chinese ink via facial and maxillary artery respectively and simultaneously. In the heads, already fixed and conserved, the arrival of the colour of the injected ink was macroscopically analyzed up to the level of the gingiva associated to the upper right incisive and canine teeth; subsequently, the heads were frozen to make them cross sections, which were analyzed mesoscopically, where the arrival of the injected ink was observed up to the level of the alveolus and pulpar cavity of the teeth under study.

The present research demonstrated that the facial artery is distributed to deep-lying tissues such as gingiva, alveolus, and pulpar cavity of the upper right incisive and canine teeth in dogs; therefore, it would be contributing to its complementary irrigation, the participation of the branches of the maxillary artery in the irrigation of these tissues, and, finally, it demonstrated that the branches of the facial and maxillary artery anastomose themselves to irrigate tissues they have in common, as the ones that were already mentioned. These results allow us knowing the origin of the revascularization of the upper and periodontal teeth in dogs, after surgical procedures that could damage some branches of maxillary artery. Due to the anatomic similarity of human being's oral cavity and the oral cavity of dogs, the already described results could be used as reference to explain the origin of the revascularization of upper and periodontal teeth, after orthognathic surgery.

KEY WORDS: 1. Facial artery; 2. Maxillary artery; 3. Alveolus; 4. Pulpar cavity. 


\section{REFERENCIAS BIBLIOGRÁFICAS}

Cheung, L. K.; Fung, S. C.; Li, T. \& Samman, N. Posterior maxillary anatomy: Implications for Le Fort I osteotomy. Int. J. oral and maxillofacial surg., 27:34251,1998

Evans, H. E. Anatomy of the dog. $3^{\text {ed. }}$ New York, W. B. Saunders company, 1993. 1113 p.

Figún, M. E. \& Garino, R. R. Anatomía Odontológica funcional y aplicada. $2^{\text {ed }}$. Buenos Aires, El Ateneo, 2002. $518 \mathrm{p}$.

Justus, T.; Chang, B. L.; Bloomquist, D. \& Ramsay, D.S. Human gingival and pulpal blood flow during healing alter Le Fort I osteotomy. J. oral and maxillofacial surg., 59(1): 2-7, 2001.

\author{
Dirección para correspondencia: \\ Dra. Cintya Borroni \\ Universidad Santo Tomás \\ $\mathcal{A} v$. Ejercito 146 \\ Santiago - CHILE \\ Email: cintyab@gmail.com
}

Recibido : 08-07-2006

Aceptado: 12-09-2006 
